\title{
REAL ASYMPTOTIC SOLUTIONS OF REAL DIFFERENTIAL EQUATIONS ${ }^{1}$
}

\author{
MONROE H. MARTIN
}

Let us consider the system of differential equations

(1) $\quad \dot{x}_{k}=\sum_{j=1}^{n} a_{k j} x_{j}+\phi_{k}\left(x_{1}, \cdots, x_{n}\right), \quad \dot{x}_{k}=\frac{d x_{k}}{d t}, \quad k=1, \cdots, n$,

in which the $a_{k j}$ denote constants and the $\phi_{k}\left(x_{1}, \cdots, x_{n}\right)$ are power series in $x_{1}, \cdots, x_{n}$ converging in a given neighborhood of the origin $x_{1}=\cdots=x_{n}=0$, and containing neither constant nor linear terms. The differential equations in the system are supposed to be real and we shall investigate real solutions

$$
x_{k}=x_{k}(t), \quad k=1, \cdots, n,
$$

which are asymptotic to the origin as $t$ tends to $+\infty$ through real values, that is, real solutions for which

$$
\lim _{t \rightarrow+\infty}\left(x_{1}^{2}+\cdots+x_{n}^{2}\right)=0 \text {. }
$$

When $n=2$ the results to which we shall be led are well known ${ }^{2}$ and may be formulated as follows:

If the characteristic constants of the matrix $\left\|a_{k j}\right\|$ in (1) are both negative and one is not an integral multiple of the other, or are conjugate complex numbers with negative real parts, all the points in a suitably restricted neighborhood of the origin lie on real solutions of (1) which are asymptotic to the origin as t tends to $+\infty$. If the characteristic constants are real and of opposite signs, a point in a suitably restricted neighborhood of the origin lies on a real solution of (1) asymptotic to the origin as $t$ tends to $+\infty$ if, and only if, it is a point of a certain real analytic arc containing the origin.

Recent dynamical investigations ${ }^{3}$ by the author require an exten-

${ }^{1}$ Presented to the Society, December 29, 1938, under the title Restricted problems in three bodies.

${ }^{2}$ See, for example, E. Picard, Traité d'Analyse, vol. 3, 1928, pp. 206-213. In connection with the case where the characteristic constants have opposite signs, cf. P. Painlevé, Gewöhnliche Differentialgleichungen; Existenz der Lösungen, Encyklopädie der mathematischen Wissenschaften, vol. 2, pt. $1^{1}$, p. 221 (footnote 116), where it is pointed out that Poincare failed to recognize the necessity of proving what is equivalent to showing that a point not on the stated analytic arc cannot lie on a solution of (1) asymptotic to the origin as $t$ tends to $+\infty$. See also E. Picard, loc. cit., p. 207.

${ }^{3}$ Not yet published. 
sion of this theorem to the cases $n>2$. That the extension is not obvious has been maintained by Bieberbach. ${ }^{4}$ The difficulty in generalizing the theorem arises when some of the characteristic constants of the matrix $\left\|a_{k j}\right\|$ are complex. The need for a proof of the existence of real solutions asymptotic to the origin when some of the characteristic constants are complex has been emphasized by Wintner. ${ }^{5}$

The general theorem to be established follows.

THEOREM. If the first $m$ of the characteristic constants $\lambda_{k}$ of the matrix $\left\|a_{k j}\right\|$ in the system (1) are negative or have negative real parts, while the remaining $n-m$ are positive or have positive real parts, if the elementary divisors of the characteristic matrix are linear, and if none of the linear commensurability relations

$$
\begin{aligned}
& \lambda_{k}=p_{1} \lambda_{1}+\cdots+p_{m} \lambda_{m}, \\
& k=1, \cdots, m ; p_{k}=0,1, \cdots ; p_{1}+\cdots+p_{m} \geqq 2,
\end{aligned}
$$

holds, a point in a suitably restricted neighborhood of the origin lies on a real solution of (1) asymptotic to the origin as $t$ tends to $+\infty$ if, and only if, it is a point of a certain real, analytic, m-dimensional manifold regular at the origin.

A companion theorem corresponding to the remaining $n-m$ characteristic constants and dealing with real solutions asymptotic to the origin as $t$ tends to $-\infty$ may obviously be formulated.

Our investigation is based on the following results ${ }^{6}$ due to Poincaré, Picard and Horn.

Assume given the system of differential equations

$$
\dot{z}_{k}=\lambda_{k} z_{k}+\phi_{k}\left(z_{1}, \cdots, z_{n}\right), \quad k=1, \cdots, n,
$$

in which $z_{k}$ denote real or complex variables, $\phi_{k}\left(z_{1}, \cdots, z_{n}\right)$ denote real or complex power series in $z_{1}, \cdots, z_{n}$, which converge in a given neighborhood of $z_{1}=\cdots=z_{n}=0$ and begin with terms of at least the second degree in $z_{1}, \cdots, z_{n}$. The constants $\lambda_{k}$ may be real or complex and it is assumed that $\lambda_{1}, \cdots, \lambda_{m}$ have negative real parts while $\lambda_{m+1}, \cdots, \lambda_{n}$ have positive real parts. In addition the constants $\lambda_{k}$ are subject to the hypothesis that none of the linear commensurability relations

$$
\lambda_{k}=p_{1} \lambda_{1}+\cdots+p_{m} \lambda_{m}, \quad k=1, \cdots, m,
$$

${ }^{4}$ L. Bieberbach, Differentialgleichungen, 3d edition, 1930, p. 113.

5 A. Wintner, Librationstheorie des restringierten Dreikörperproblems, Mathematische Zeitschrift, vol. 32 (1930), pp. 661-662.

${ }^{6}$ E. Picard, op. cit., pp. 17-21, and pp. 187-188; J. Horn, Gewöhnliche Differentialgleichungen beliebiger Ordnung, 1905, pp. 319-325. For (5) in the text above, see page 321 of Horn's book. 
holds, where $p_{1}, \cdots, p_{m}$ denote non-negative integers for which $p_{1}+\cdots+p_{m} \geqq 2$.

If $C_{1}, \cdots, C_{m}$ denote $m$ arbitrary real or complex constants, it is possible to find power series for $z_{1}, \cdots, z_{n}$ in the arguments

$$
u_{1}=C_{1} e^{\lambda_{1} t}, \cdots, u_{m}=C_{m} e^{\lambda_{m} t},
$$

which converge for sufficiently small absolute values of these arguments and which satisfy the differential equations. These power series have the form

$$
\begin{aligned}
& z_{j}=u_{j}+P_{j}\left(u_{1}, \cdots, u_{m}\right), \quad j=1, \cdots, m, \\
& z_{k}=P_{k}\left(u_{1}, \cdots, u_{m}\right), \quad k=m+1, \cdots, n,
\end{aligned}
$$

where the power series $P_{1}, \cdots, P_{n}$ begin with terms of at least the second degree in $u_{1}, \cdots, u_{m}$.

The complex characteristic constants of the real matrix $\left\|a_{k j}\right\|$ necessarily occur in conjugate pairs and we arrange the notation so that

$$
\begin{aligned}
\bar{\lambda}_{2 \nu_{1}-1} & =\lambda_{2 \nu_{1}}, & \nu_{1}=1, \cdots, j_{0}, \\
\bar{\lambda}_{\nu_{2}} & =\lambda_{\nu_{2}}<0, & \nu_{2}=2 j_{0}+1, \cdots, m, \\
\bar{\lambda}_{m+2 \nu_{3}-1} & =\lambda_{m+2 \nu_{3}}, & \nu_{3}=1, \cdots, k_{0}, \\
\bar{\lambda}_{\nu_{4}} & =\lambda_{\nu_{4}}>0, & \nu_{4}=m+2 k_{0}+1, \cdots, n,
\end{aligned}
$$

where $j_{0}, k_{\mathrm{G}}$ denote integers such that $0 \leqq 2 j_{0} \leqq m, 0 \leqq 2 k_{0} \leqq n-m$. The $\nu_{1}, \nu_{2}, \nu_{3}, \nu_{4}$ will always be used in the sequel in the same sense as employed here. Thus, to indicate the real and imaginary parts of the complex characteristic constants, we shall write

$$
\begin{aligned}
\lambda_{2 \nu_{1}-1} & =\alpha_{\nu_{1}}+i \beta_{\nu_{1}}, & \lambda_{2 \nu_{1}} & =\alpha_{\nu_{1}}-i \beta_{\nu_{1}}, \\
\lambda_{m+2 \nu_{3}-1} & =\alpha_{m+\nu_{3}}+i \beta_{m+\nu_{3}}, & \lambda_{m+2 \nu_{3}} & =\alpha_{m+\nu_{3}}-i \beta_{m+\nu_{3}} .
\end{aligned}
$$

From the theory of linear transformations it follows that there exists a real linear transformation with nonvanishing determinant transforming the variables $x_{1}, \cdots, x_{n}$ into the variables $y_{1}, \cdots, y_{n}$ and the system (1) of differential equations into the system

$$
\begin{aligned}
\dot{y}_{2 \nu_{1}-1} & =\alpha_{\nu_{1}} y_{2 \nu_{1}-1}-\beta_{\nu_{1}} y_{2 \nu_{1}}+\Phi_{2 \nu_{1}-1}\left(y_{1}, \cdots, y_{n}\right), \\
\dot{y}_{2 \nu_{1}} & =\beta_{\nu_{1}} y_{2 \nu_{1}-1}+\alpha_{\nu_{1}} y_{2 \nu_{1}}+\Phi_{2 \nu_{1}}\left(y_{1}, \cdots, y_{n}\right), \\
\dot{y}_{\nu_{2}} & =\lambda_{\nu_{2}} y_{\nu_{2}}+\Phi_{\nu_{2}}\left(y_{1}, \cdots, y_{n}\right), \\
\dot{y}_{m+2 \nu_{3}-1} & =\alpha_{m+\nu_{3}} y_{m+2 \nu_{3}-1}-\beta_{m+\nu_{3}} y_{m+2 \nu_{3}}+\Phi_{m+2 \nu_{3}-1}\left(y_{1}, \cdots, y_{n}\right), \\
\dot{y}_{m+2 \nu_{3}} & =\beta_{m+\nu_{3}} y_{m+2 \nu_{3}-1}+\alpha_{m+\nu_{3}} y_{m+2 \nu_{3}}+\Phi_{m+2 \nu_{3}}\left(y_{1}, \cdots, y_{n}\right), \\
\dot{y}_{\nu_{1}} & =\lambda_{\nu_{4}} y_{\nu_{4}}+\Phi_{\nu_{4}}\left(y_{1}, \cdots, y_{n}\right),
\end{aligned}
$$


where $\Phi_{1}, \cdots, \Phi_{n}$ denote real power series in $y_{1}, \cdots, y_{n}$ beginning with terms of at least the second degree in these variables.

If complex variables $z_{1}, \cdots, z_{n}$ be introduced by means of the equations

$$
\begin{array}{rlrl}
z_{2 \nu_{1}-1} & =y_{2 \nu_{1}-1}+i y_{2 \nu_{1}}, \quad z_{2 \nu_{1}}=y_{2 \nu_{1}-1}-i y_{2 \nu_{1}}, & z_{\nu_{2}} & =y_{\nu_{2}}, \\
z_{m+2 \nu_{3}-1} & =y_{m+2 \nu_{3}-1}+i y_{m+2 \nu_{3}}, z_{m+2 \nu_{3}}=y_{m+2 \nu_{3}-1}-i y_{m+2 \nu_{3}}, & z_{\nu_{4}}=y_{\nu_{4}},
\end{array}
$$

the differential equations ( 7 ) take the simple form

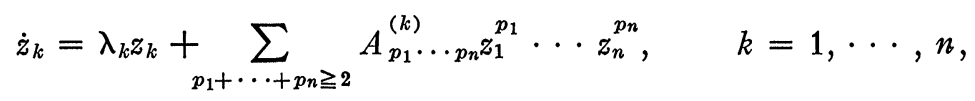

where

$$
\bar{A}_{p_{1} \cdots p_{n}}^{(k)}=A_{q_{1}}^{(j)} \cdots q_{n} \quad \text { whenever } \bar{\lambda}_{k}=\lambda_{j},
$$

and $q_{1}, \cdots, q_{n}$ is the permutation of $p_{1}, \cdots, p_{n}$ obtained by interchanging every pair $p_{k}, p_{l}$ for which $k$ and $l$ are such that $\bar{\lambda}_{k}=\lambda_{l}$.

It is apparent from (8) that a solution of (7) will be real if, and only if, the corresponding solution of (9) satisfies the condition

$$
\bar{z}_{k}=z_{j} \quad \text { whenever } \bar{\lambda}_{k}=\lambda_{j},
$$

and we shall now construct solutions of (9) satisfying these conditions.

The following method was suggested by my colleague E. Titt and I have adopted it in preference to my own as less cumbersome.

Referring to the definition of $\nu_{1}, \nu_{2}, \nu_{3}, \nu_{4}$ in (6), it follows from (10) that if

$$
z_{2 \nu_{1}-1}, z_{2 \nu_{1}}, z_{\nu_{2}}, z_{m+2 \nu_{3}-1}, z_{m+2 \nu_{3}}, z_{\nu_{4}}
$$

denotes a set of $n$ real or complex functions of $t$ which is a solution of (9), then the set

$$
\bar{z}_{2 \nu_{1}}, \bar{z}_{2 \nu_{1}-1}, \bar{z}_{\nu_{2}}, \bar{z}_{m+2 \nu_{3}}, \bar{z}_{m+2 \nu_{3}-1}, \bar{z}_{\nu_{4}} \text {, }
$$

obtained from (12) by replacing $z_{k}(t)$ by $\bar{z}_{j}(t)$ whenever $\bar{\lambda}_{k}=\lambda_{j}$ $(1 \leqq k \leqq j \leqq n)$, also represents a solution of (9). Accordingly a solution of (7) obtained from (8), will be real if, and only if, the two solutions (12) and (13) of (9) are identical.

The results of Poincaré and Picard apply to the system (9) and we write the solution (5) in the form

$$
z_{k}=\sum_{k_{1}+\cdots+k_{m} \geqq 1} C_{k_{1} \cdots k_{m}}^{(k)} u_{1}^{k_{1}} \cdots u_{m}^{k_{m}}
$$


where we have, of course, when $k_{1}+\cdots+k_{m}=1$,

$$
C_{k_{1} \cdots k_{m}}^{(j)}=1, \quad k_{1}=0, \cdots, k_{j}=1, \cdots, k_{m}=0 ; j=1, \cdots, m,
$$

the $C_{\boldsymbol{k}_{1}}^{(k)} \ldots k_{m}$ remaining, for which $k_{1}+\cdots+k_{n}=1, k=1, \cdots, n$, being zero. Choose the arbitrary constants $C_{1}, \cdots, C_{m}$ in (4) so that

$$
\bar{C}_{p}=C_{q} \quad \text { whenever } \bar{\lambda}_{p}=\lambda_{q},(p \leqq q \leqq m),
$$

and therefore

$$
\bar{u}_{p}=u_{q} \quad \text { whenever } \bar{\lambda}_{p}=\lambda_{q},(p \leqq q \leqq m) .
$$

Selecting the solution (14) for the solution in (12), we shall prove that it is identical with the solution (13). Upon substituting (14) in (9) and equating coefficients of $u_{1}^{k_{1}} \cdots u_{m}^{k_{m}}$ it may be verified that $C_{k_{1} \cdots k_{m}}^{(k)}$, for $k_{1}+\cdots+k_{m}=r(r \geqq 2)$ and $k$ fixed, is expressible as a polynomial in the $C_{k_{1}}^{(k)} \ldots k_{m}$ with $k_{1}+\cdots+k_{m}<r$ and $k=1,2, \cdots, n$. These recurrence equations determine the $C_{k_{1}}^{(k)} \ldots k_{m}$ uniquely, once the coefficients of the linear terms in $u_{1}, \cdots, u_{n}$ of (14) are given. Taking the coefficients of the linear terms in $u_{1}, \cdots, u_{m}$ of (12) as given by (15), it follows from (17) that they are identical with the coefficients of the linear terms in $u_{1}, \cdots, u_{m}$ of (13), and the two solutions are therefore identical.

Accordingly, the constants $C_{j}$ in (4) being chosen subject to (16) and the complex solution (14) of (9) inserted in (8), a real solution of (7) is obtained when equations (8) are solved for $y_{1}, \cdots, y_{n}$.

If we write

$$
u_{2 \nu_{1}-1}=v_{2 \nu_{1}-1}+i v_{2 \nu_{1}}, \quad u_{2 \nu_{1}}=v_{2 \nu_{1}-1}-i v_{2 \nu_{1}}, \quad u_{\nu_{2}}=v_{\nu_{2}},
$$

the afore-mentioned real solution of (7) takes the form

$$
\begin{aligned}
& y_{j}=v_{j}+Q_{j}\left(v_{1}, \cdots, v_{m}\right), \quad j=1, \cdots, m, \\
& y_{k}=Q_{k}\left(v_{1}, \cdots, v_{m}\right), \quad k=m+1, \cdots, n,
\end{aligned}
$$

where $Q_{1}, \cdots, Q_{n}$ denote real power series beginning with terms of at least the second degree in the real variables $v_{1}, \cdots, v_{m}$, and converging for sufficiently small $\left|v_{1}\right|, \cdots,\left|v_{m}\right|$. If we let

$$
C_{2 \nu_{1}-1}=a_{\nu_{1}}+i b_{\nu_{1}}, \quad C_{2 \nu_{1}}=a_{\nu_{1}}-i b_{\nu_{1}}, \quad C_{\nu_{2}}=a_{\nu_{2}},
$$

it is readily seen that

$$
\begin{aligned}
v_{2 \nu_{1}-1} & =e^{\alpha \nu_{1} t}\left(a_{\nu_{1}} \cos \beta_{\nu_{1}} t-b_{\nu_{1}} \sin \beta_{\nu_{1}} t\right), \\
v_{2 \nu_{1}} & =e^{\alpha \nu_{1} t}\left(a_{\nu_{1}} \sin \beta_{\nu_{1}} t+b_{\nu_{1}} \cos \beta_{\nu_{1}} t\right), \quad v_{\nu_{2}}=a_{\nu_{2}} e^{\lambda \nu_{2} t} .
\end{aligned}
$$


The constants $a_{\nu_{1}}, b_{\nu_{1}}, a_{\nu_{2}}$ are real and arbitrary. It therefore follows from (19) and (20) that there exists a real, analytic, $m$-dimensional manifold of solutions of $(7)$ in a sufficiently small neighborhood of the origin $y_{1}=\cdots=y_{n}=0$. This $m$-dimensional manifold is regular at the origin, and, since $\alpha_{\nu_{1}}, \lambda_{\nu_{2}}$ are negative, any one of its points lies on a real solution of (1) asymptotic to the origin as $t$ tends to $+\infty$.

We shall now show that a sufficiently small neighborhood of the origin $y_{1}=\cdots=y_{n}=0$ contains no points on real solutions of (7) asymptotic to the origin as $t$ tends to $+\infty$ other than the points of the above manifold.

Solving the first $m$ equations in (19) for $v_{1}, \cdots, v_{m}$ and substituting these expressions in the remaining $n-m$ equations, we find the equations of the $m$-dimensional manifold in non-parametric form, namely

$$
y_{k}=Q_{k}^{*}\left(y_{1}, \cdots, y_{m}\right), \quad k=m+1, \cdots, n,
$$

where $Q_{k}^{*}$ denote power series beginning with terms of the second degree at least in $y_{1}, \cdots, y_{m}$ and converging for sufficiently small $\left|y_{1}\right|, \cdots,\left|y_{m}\right|$.

Let us introduce new coordinates $\eta_{1}, \cdots, \eta_{n}$ by means of the equations

$$
\begin{array}{lr}
\eta_{j}=y_{j}, & j=1, \cdots, m, \\
\eta_{k}=y_{k}-Q_{k}^{*}\left(y_{1}, \cdots, y_{m}\right), & k=m+1, \cdots, n .
\end{array}
$$

The differential equations (7) retain their form after transformation, the $y$ 's being replaced, of course, by $\eta$ 's. The coefficients of the linear terms are unaltered and the power series $\Phi_{1}, \cdots, \Phi_{n}$ become power series in $\eta_{1}, \cdots, \eta_{n}$ which will be denoted by $\Phi_{1}^{*}, \cdots, \Phi_{n}{ }^{*}$ respectively.

The last $n-m$ of the transformed equations must be satisfied when we put $\eta_{m+1}=\cdots=\eta_{n}=0$, and therefore

$$
\Phi_{k}^{*}=\sum_{l=m+1}^{n} \Phi_{k \imath} \eta_{l}, \quad k=m+1, \cdots, n,
$$

where $\Phi_{k l}$ denote power series in $\eta_{1}, \cdots, \eta_{n}$ without constant terms. Multiplying the last $n-m$ transformed equations by $\eta_{m+1}, \cdots, \eta_{n}$, respectively and adding, there results

$$
\sum_{k=m+1}^{n} \eta_{k} \dot{\eta}_{k}=\sum_{k=m+1}^{n} \alpha_{k}{ }^{2} \eta_{k}+\sum_{k=m+1}^{n} \sum_{l=m+1}^{n} \Phi_{k} \eta_{k} \eta_{l},
$$

where we have employed (22) to substitute for $\Phi_{k}^{*}$. Writing

$$
\rho^{2}=\eta_{m+1}^{2}+\cdots+\eta_{n}^{2}, \quad \mu_{k}=\eta_{k} / \rho, \quad k=m+1, \cdots, n,
$$


in the above equation and dividing by $\rho^{2}$, we find

$$
\dot{\rho} / \rho=\sum_{k=m+1}^{n} \alpha_{k} \mu_{k}^{2}+\sum_{k=m+1}^{n} \sum_{l=m+1}^{n} \Phi_{k} \mu_{k} \mu_{l} .
$$

Let $\alpha^{*}=\min \left\{\alpha_{m+1}, \cdots, \alpha_{n}\right\}$ and $\Phi^{*}=\max \left\{\left|\Phi_{k l}\right|\right\}$, where $\Phi^{*}=$ $\Phi^{*}\left(\eta_{1}, \cdots, \eta_{n}\right)$ tends to zero as the point $\left(\eta_{1}, \cdots, \eta_{n}\right)$ tends towards the origin $\eta_{1}=\cdots=\eta_{n}=0$. Now $\mu_{m+1}^{2}+\cdots+\mu_{n}^{2}=1$, so that we have

$$
\left|\sum_{k=m+1}^{n} \sum_{l=m+1}^{n} \Phi_{k} \mu_{k} \mu_{l}\right| \leqq(n-m) \Phi^{*},
$$

and hence from (23) we see that

$$
\dot{\rho} / \rho \geqq \alpha^{*}-(n-m) \Phi^{*}, \quad \quad \alpha^{*}>0 .
$$

If possible let $y_{k}=y_{k}(t)$ be a real solution of (7) which is asymptotic to the origin $y_{1}=\cdots=y_{n}=0$ as $t$ tends to $+\infty$, yet for which there is no $\bar{t}$ such that the solution lies in the $m$-dimensional manifold (21) for $t>\bar{t}$.

Employing $\eta_{1}, \cdots, \eta_{n}$ as coordinates, we should then have a real solution $\eta_{k}=\eta_{k}(t)$ of the transformed differential equations with the property that to any positive. number $\epsilon$ there corresponds a $t_{\epsilon}>0$ such that we have

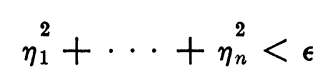

for all $t>t_{\epsilon}$. In addition there must exist a $t_{0}>t_{\epsilon}$ which is such that on writing $\eta_{k}^{0}=\eta_{k}\left(t_{0}\right), \quad \rho_{0}^{2}=\eta_{m+1}^{0^{2}}+\cdots+\eta_{n}^{0^{2}}(k=1, \cdots, n)$, we have $\rho_{0}>0$. Since $\Phi^{*}$ is arbitrarily small in the neighborhood of the origin of $\eta_{1}=\cdots=\eta_{n}=0$, it is possible to choose $\epsilon$ at the outset so small that

$$
\Phi^{*}=\Phi^{*}\left(\eta_{1}(t), \cdots, \eta_{n}(t)\right)<\alpha^{*} / 2(n-m) \quad \text { for } t>t_{\epsilon} .
$$

Inequality (24) then becomes $\dot{\rho} / \rho>\alpha^{*} / 2$ for $t>t_{\epsilon}$. Integrating from $t_{0}$ to $t\left(t>t_{0}\right)$, we find

$$
\rho>\rho_{0} e^{\left(\alpha^{*} / 2\right)\left(t-t_{0}\right)},
$$

which, since $\alpha^{*}>0$, implies a contradiction to (25).

Since the transformation from the system (1) to the system (7) was a real linear transformation with nonvanishing determinant the theorem has now been established.

UNIVERSITY OF MARYLAND 